\title{
Port-site metastasis as a primary complication following retroperitoneal laparoscopic radical resection of renal pelvis carcinoma or nephron-sparing surgery: A report of three cases and review of the literature
}

\author{
NING WANG ${ }^{1,2}$, KAI WANG $^{3}$, DACHUAN ZHONG ${ }^{3}$, XIA LIU $^{1}$, JI SUN $^{3}$, \\ LIANXIANG LIN ${ }^{3}$, LINNA GE ${ }^{4}$ and BO YANG ${ }^{1}$ \\ ${ }^{1}$ Department of Urology, The Second Affiliated Hospital of Dalian Medical University, Dalian, Liaoning 116027; \\ ${ }^{2}$ Hangzhou Tourism Vocational School, Hangzhou, Zhejiang 311200; ${ }^{3}$ Department of Urology, \\ Zhejiang Xiaoshan Hospital, Hangzhou, Zhejiang 311202; ${ }^{4}$ Department of Radiology, \\ The General Hospital of Jixi Mining Group, Jixi, Heilongjiang 158100, P.R. China
}

Received February 8, 2015; Accepted March 1, 2016

DOI: $10.3892 / \mathrm{ol} .2016 .4541$

\begin{abstract}
The present study reports the clinical data of two patients with renal pelvis carcinoma and one patient with renal carcinoma who developed port-site metastasis following retroperitoneal laparoscopic surgery. The current study aimed to identify the cause and prognosis of the occurrence of port-site metastasis subsequent to laparoscopic radical resection of renal pelvis carcinoma and nephron-sparing surgery. Post-operative pathology confirmed the presence of high-grade urothelial cell carcinoma in two patients and Fuhrman grade 3 renal clear cell carcinoma in one patient. Port-site metastasis was initially detected 1-7 months post-surgery. The two patients with renal pelvis carcinoma succumbed to the disease 2 and 4 months following the identification of the port-site metastasis, respectively, whereas the patient with renal carcinoma survived with no disease progression during the targeted therapy period. The occurrence of port-site metastasis may be attributed to systemic and local factors. Measures to reduce the development of this complication include strict compliance with the operating guidelines for tumor surgery, avoidance of air leakage at the port-site, complete removal of the specimen with an impermeable bag, irrigation of the laparoscopic instruments and incisional wound with povidone-iodine when necessary, and enhancement of the body's immunity. Close post-operative follow-up observation for signs of recurrence
\end{abstract}

Correspondence to: Professor Bo Yang, Department of Urology, The Second Affiliated Hospital of Dalian Medical University, 467 Zhongshan Road, Shahekou, Dalian, Liaoning 116027, P.R. China

E-mail: dymnyb@hotmail.com

Key words: retroperitoneal laparoscopy, renal pelvis carcinoma, renal carcinoma, port-site metastasis or metastasis is essential, and systemic chemotherapy may be required in patients with high-grade renal pelvis carcinoma and renal carcinoma in order to prolong life expectancy.

\section{Introduction}

Post-laparoscopic occurrence of port-site metastasis refers to tumor foci either localized at single or multiple locations under the skin or in the scar tissue of the abdominal wall adjacent to the port (1). Port-site metastasis is a rare complication that may occur following laparoscopic surgery for malignant tumors of the urinary system, with an incidence of $0.09-0.73 \%$ of all patients who undergo laparoscopic surgery for urological malignancies (2,3). Previous studies have reported $\sim 50$ cases of abdominal wall implantation metastasis following surgical resection of malignant tumors of the urinary system (4), of which, 9 cases occurred following surgical resection of renal carcinoma $(5-18,15)$. Thus, this indicates that the occurrence of port-site metastasis subsequent to laparoscopic radical resection of renal carcinoma and nephron-sparing surgery is relatively rare. Although previous studies have reported the pathogenesis, risk factors and prevention of port-site metastasis following laparoscopic radical resection of renal carcinoma and nephron-sparing surgery $(14,15)$, few studies have reported the mechanism and prognosis of port-site metastasis occurring in the case of renal pelvis carcinoma $(19,20)$. The present study reports the clinical data of two patients with renal pelvis carcinoma and one patient with renal carcinoma who developed port-site metastasis following retroperitoneal laparoscopic surgery with the aim of identifying the cause and prognosis of port-site metastasis occurring in such circumstances. In addition, a review of the literature is conducted in the present study.

\section{Case report}

Case 1. A 71-year-old male was admitted to The Second Affiliated Hospital of Dalian Medical University (Dalian, China) 
August 28, 2009, presenting with hematuria. Computed tomography (CT; SOMATOM Definition AS 64; Siemens AG, Munich, Germany) urography was performed following patient admission, which revealed fluid collection in the left renal pelvis and left ureter. A cystoscopy was subsequently performed, which identified blood ejection at the orifice of the left ureter. Three consecutive urine exfoliative cytology tests suggested the presence of urothelial cell carcinoma. Retroperitoneal laparoscopic radical nephroureterectomy and bladder cuffing resection, including the ureteral orifice, was performed. The specimen was completely excised, enveloped in an impermeable specimen bag and pulled out through external rectus incision. Formalin-fixed paraffin-embedded tissues (FFPET) were cut into 4- $\mu \mathrm{m}$ sections and stained with hematoxylin-eosin (HE) to evaluate the cell pattern. The sections were scanned under a light microscope and images were captured at a magnification of x200. Post-operative pathology revealed tumor cells in papillary and solid nests with a disordered arrangement and loss of polarity, which confirmed the diagnosis of high-grade infiltrative urothelial cell carcinoma of the left renal pelvis and ureter. Approximately 7 months after the initial surgery, a painless hard mass was detected under the lumbar skin. A CT scan performed at the hospital revealed a subcutaneous metastatic tumor in the left ilium measuring $\sim 2.0 \mathrm{~cm}$ in size. Left abdominal wall tumor resection was subsequently performed. The aforementioned tissue preparation method for histopathological examination was used. The post-operative pathology revealed tumor cells with large nucleoli and the formation of cancerous cell nests in the fibrous cords, which confirmed the diagnosis of a metastatic malignant tumor (Fig. 1). At 2 weeks post-resection, a rapidly progressive tumor was detected in the left lumbar region, which was confirmed by emission CT as multiple bone metastases. Abdominopelvic CT suggested the presence of liver metastasis. The patient eventually succumbed to the disease despite undergoing gemcitabine plus cisplatin (GC) chemotherapy [3 cycles of gemcitabine $\left(1,000 \mathrm{mg} / \mathrm{m}^{2}\right)$ on days 1,8 and 15 plus cisplatin $\left(70 \mathrm{mg} / \mathrm{m}^{2}\right)$ on day 2 every 28 days, each administered via intravenous drip].

Case 2. A 47-year-old female was admitted to The Second Affiliated Hospital of Dalian Medical University on July 4, 2013, due to the previous detection of a tumor in the urinary bladder during a physical examination. The tumor was removed via transurethral resection, and the tissue resected was observed to be deep to the muscular layer. Post-operative pathology confirmed the diagnosis of low-grade urothelial cell carcinoma. The patient received regular pirarubicin perfusion chemotherapy (20 mg THP dissolved in $40 \mathrm{ml} 5 \%$ dextrose was administered intravesically once a week for 6 weeks, then once a month) post-operatively. Blood ejection was detected at the right ureter 10 months after the initial surgery during a cystoscopic examination at the clinic of the hospital. A lower abdominal contrast-enhanced magnetic resonance imaging (MAGNETOM Verio 3T; Siemens AG) scan revealed a tumor in the upper pole of the right kidney measuring $\sim 4.0 \times 3.6 \mathrm{~cm}$ in size with uneven enhancement. Three consecutive urine exfoliative cytology tests suggested the presence of urothelial cell carcinoma. Retroperitoneal laparoscopic radical nephroureterectomy and bladder cuffing resection, including the ureteral orifice, was consequently performed. The specimen was completely excised, enveloped in an impermeable specimen bag and pulled out through external rectus incision. FFPET with HE staining were observed in 4- $\mu \mathrm{m}$ sections by light microscope under magnification of x200. Post-operative pathology confirmed the diagnosis of high-grade infiltrative urothelial cell carcinoma of the right renal pelvis. Microscopically, the tumor was composed of high-grade malignant urothelial cells. Numerous pleomorphic, giant and multinucleated cells with one or more prominent nucleoli were also observed. The tumor invaded the renal parenchyma with tumor emboli detected in the venous lumen; however, no tumor tissue was observed in the vascular stump of the renal hilum and the right ureteral stump. Multiple hard, painless masses were detected at the right abdominal port-site and the right lumbodorsal port-site within 1 month of the initial surgery. An abdominal CT scan performed at the hospital revealed metastatic tumors in the right abdominal wall. Pathological biopsy of these two sites demonstrated cancer cells distributed in small masses in the striated muscle, which were consistent with an urothelial origin. The aforementioned tissue preparation method for histopathological examination was used; microscopically, the carcinoma cells were arranged in the solid nest-like distribution with a large deeply-dyed nucleolus and evident pleomorphism (Fig. 2). The patient stopped any further treatment and eventually succumbed to the disease.

Case 3. A 68-year-old male was admitted to The Second Affiliated Hospital of Dalian Medical University on September 18, 2013, due to the detection of a tumor in the right kidney during a previous physical examination. The patient had a 13-year history of hypertension. Following regular administration of amlodipine, the blood pressure of the patient fluctuated between 130 and $90 \mathrm{mmHg}$. The patient also had a history of renal syndrome for 5 years, for which no regular treatment was administered. An abdominal CT scan performed following admission detected a space-occupying lesion in the middle portion of the right kidney, measuring $\sim 3.0 \times 4.0 \mathrm{~cm}$ in size. A laparoscopic nephron-sparing nephrectomy was performed, and the tumor was removed completely via a specimen bag. With the method mentioned in Case 1, the tumor tissue was managed for histopathological examination. Post-operative pathology confirmed the diagnosis of Fuhrman grade 3 (21) renal clear cell carcinoma; tumor cells exhibited clear cytoplasm and a sharply outlined cell membrane, with alveolar nests and tightly packed tubules. The tumor involved the renal capsule, but no cancer tissue was identified in the cut edge. At 9 months after the initial surgery, the patient complained of right lumbodorsal and abdominal pain. A CT plain scan was performed, which identified multiple soft-tissue intensity nodular shadows in the diaphragmatic muscle in the posterior of the right kidney, which exhibited a circular enhancement and were suspected to be metastatic tumors. CT-guided pathological biopsy of the abdominal wall tumors resulted in the diagnosis of a metastatic lesion, which was observed using a light microscope. The FFPET showed a clear cell pattern that was identical, histologically, to the previous renal cell carcinoma (Fig. 3). Sunitinib targeted therapy (50 mg daily, administered orally) was administered, but was discontinued 2 weeks after treatment initiation due to bone marrow suppression. The condition of the patient is stable at present. 
A

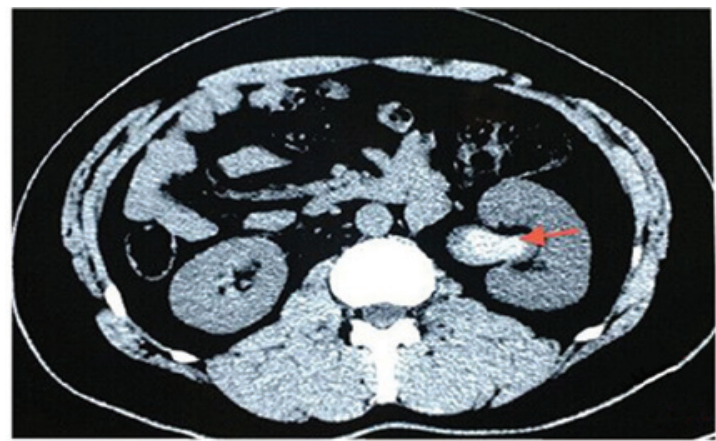

C

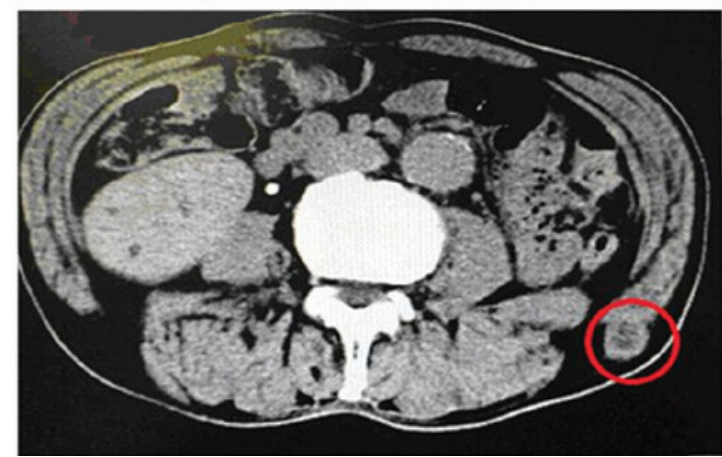

B

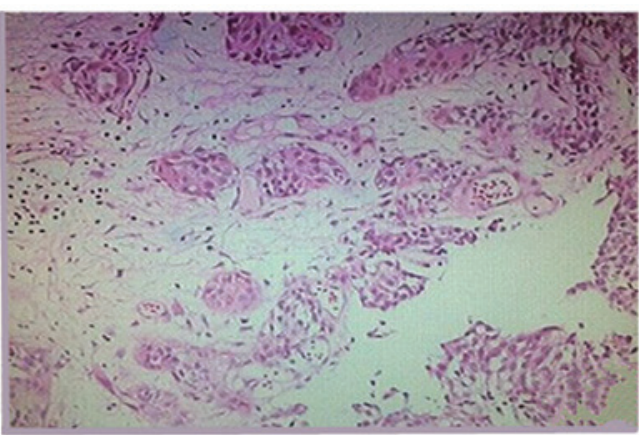

D

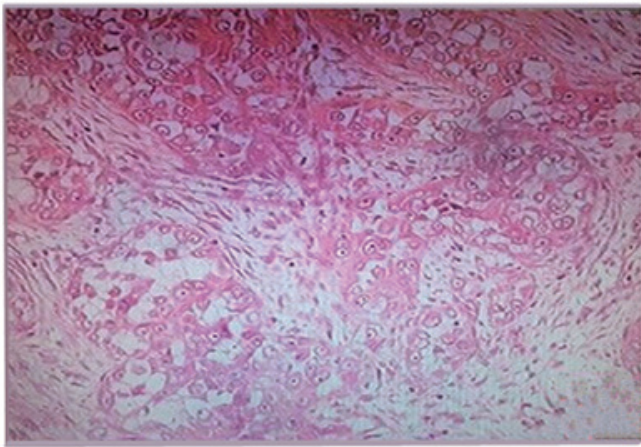

Figure 1. Images obtained from Case 1. (A) CT urography detected fluid collection and a homogeneously-enhanced mass in the left renal pelvis (arrow). (B) Pathology confirmed a diagnosis of high-grade, infiltrative, left renal urothelial cell carcinoma with cells forming nests with a high nuclear cytoplasmic ratio (staining, H\&E; magnification, x200). (C) CT revealed a subcutaneous tumor in the left ilium (red circle). (D) Pathology confirmed muscle invasion by the metastatic malignant tumor, which presented with a high-grade nuclear cytoplasmic ratio (staining, H\&E; magnification, x200). CT, computed tomography; H\&E, hematoxylin and eosin.

A

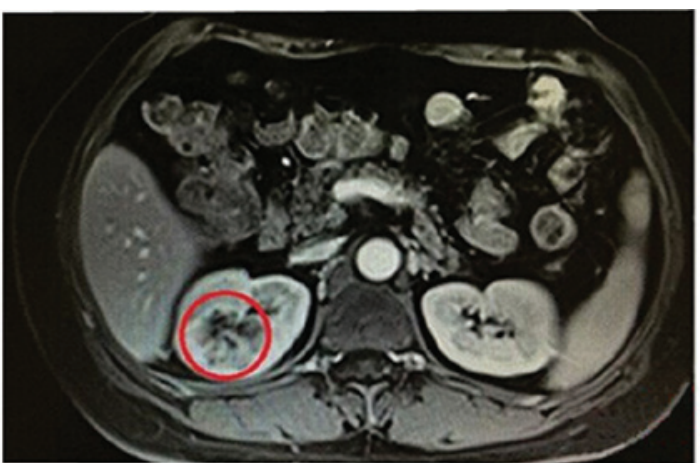

C

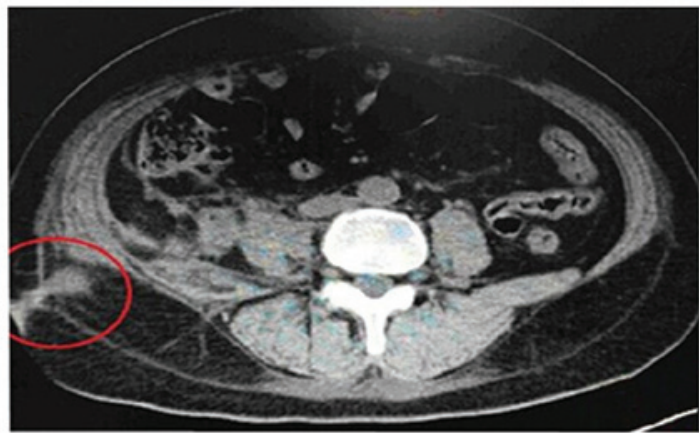

B

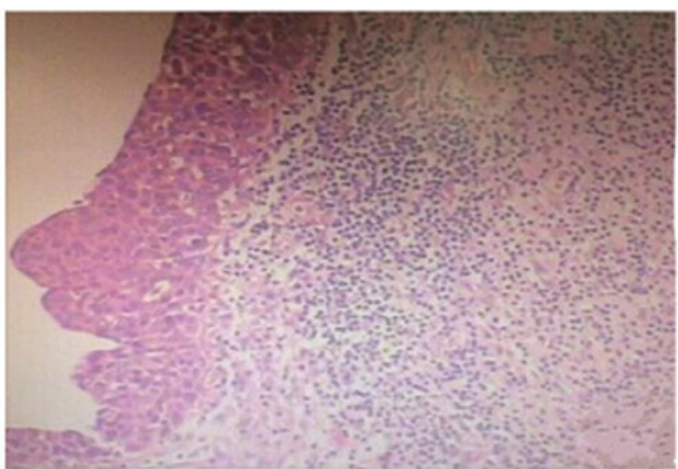

D

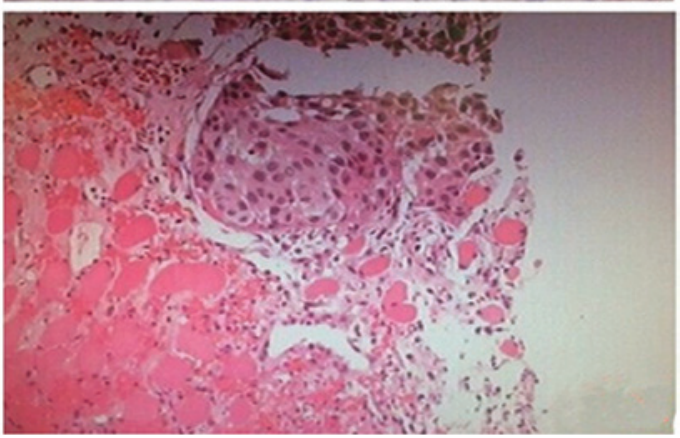

Figure 2. Images obtained from Case 2. (A) CT revealed a tumor with uneven enhancement (red circle) in the right renal pelvis. (B) Pathology confirmed a diagnosis of high-grade, infiltrative urothelial cell neoplasm with enlarged nuclei (staining, H\&E; magnification, x200). (C) CT demonstrated the presence of a metastatic tumor (red circle). (D) Pathology of a tumor biopsy showing that the muscle invasion was consistent with urothelial origin (staining, H\&E; magnification, x200). CT, computed tomography; H\&E, hematoxylin and eosin.

\section{Discussion}

Since the first case of successful laparoscopic nephrectomy was reported in 1991 (16), minimally invasive surgery has been increasingly employed for the resection and lymph node clearance of urinary malignant tumors (22-24). The therapeutic outcomes of laparoscopic surgery and open surgery are similar in the treatment of tumors $(25,26)$. The development of port-site 
A

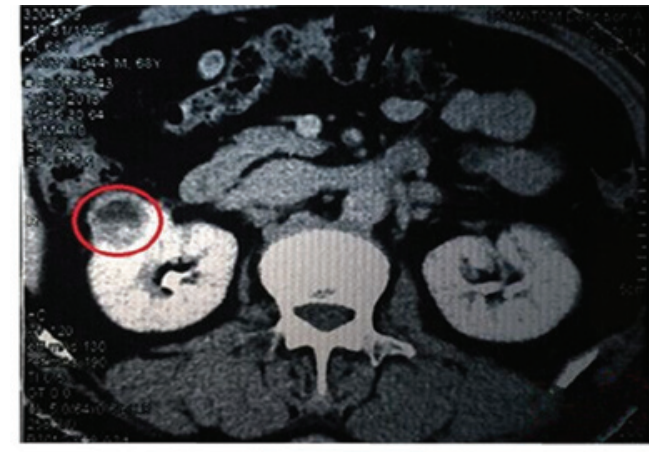

C

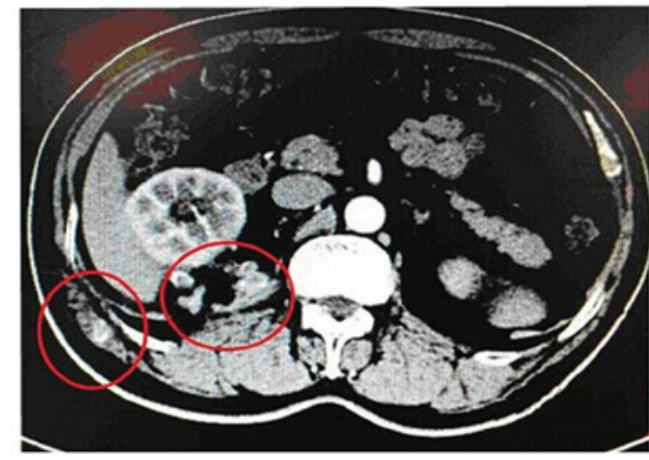

B

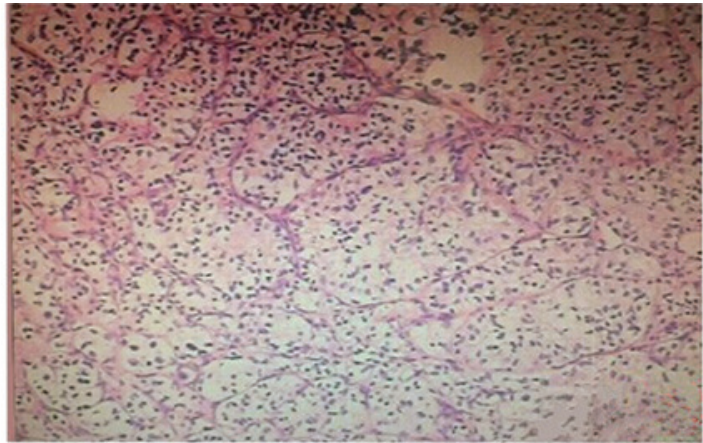

D

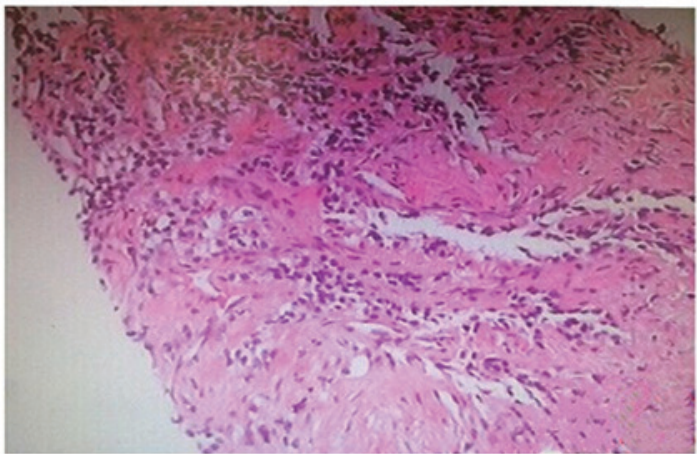

Figure 3. Images obtained from Case 3. (A) CT detected a tumor (red circle) in the right kidney. (B) Pathology confirmed a diagnosis of Fuhrman grade 3 RCC presenting with optically-clear cytoplasm and a sharply-outlined cell membrane, in addition to alveolar nests and tightly packed tubules (staining, H\&E; magnification, x200). (C) CT demonstrated metastatic tumors (red circles) in the retroperitoneal space and abdominal wall with mild- to moderate-enhancement. (D) Pathology of a tumor biopsy of the abdominal wall tumors confirmed a diagnosis of clear cell RCC with marked tumors nested in muscle tissue (staining, H\&E; magnification, x200). CT, computed tomography; RCC, renal cell carcinoma; H\&E, hematoxylin and eosin.

metastasis following laparoscopic tumor resection is rare, and the cause remains elusive $(15,17,18)$. In 1994, Stolla et al (27) reported the first case of abdominal wall port-site metastasis developing subsequent to laparoscopic pelvic lymph node clearance in a patient with bladder urothelial cell carcinoma. However, Micali et al (3) did not identify a single case of port-site metastasis in a retrospective review of the available clinical data of 2,604 patients that underwent radical nephrectomies. Therefore, the incidence of port-site metastasis remains unclear.

With regards to the incidence of local tumor dissemination following laparoscopic resection of malignant tumors, gynecological journals report an incidence of $\sim 5 \%$, while oncology journals report an incidence of $\sim 4 \%$ for colorectal cancer. The 3 cases of port-site metastasis discussed in the present study accounted for an incidence of $\sim 1.5 \%$ of all patients treated for urological malignancies in The Second Affiliated Hospital of Dalian Medical University. Certain researchers have argued that the incidence of port-site metastasis following laparoscopic radical resection of renal carcinoma is variable, and the incidence may be as high as $21 \%$ (28). However, the majority of researchers consider that port-site metastasis is rare, and the incidence is $<1 \%$ (2). In a previous study, clinical data was collected from 19 urosurgery centers, involving 10,912 patients who underwent laparoscopic resection for urinary malignant tumors (3). It was reported that only 13 cases $(0.1 \%)$ developed post-operative tumor metastasis, including 10 cases of port-site metastasis and 3 cases of retroperitoneal dissemination (3). These 13 cases included accidental detection of urothelial cell carcinoma following laparoscopic nephrectomy in 4 cases, laparoscopic nephroureterectomy for urothelial cell carcinoma in 3 cases, laparoscopic resection of adrenal metastatic cancer in 4 cases, laparoscopic pelvic lymph node clearance for penile squamous cell carcinoma in 1 case and retroperitoneal lymph node clearance for carcinoma of testis in 1 case. However, Micali et al (3) analyzed 2,604 cases of laparoscopic radical resection of renal carcinoma and did not identify any case of port-site metastasis.

Certain researchers have maintained that it is important to clarify the cause of tumor metastasis, since the prognosis of port-site metastasis is unclear (29). The following hypotheses have been largely supported as the possible causes of laparoscopic port-site metastasis: i) Biological invasiveness of tumors; ii) local traumatic factors; iii) host immune response; and iv) laparoscopic surgical procedures $(15,30)$. Based on these possible causes, experience of the 3 present cases of port-site implantation metastasis and review of the literature, the conclusions of the current study are discussed below.

Port-site metastasis and tumor recurrence are attributable to tumor invasiveness, which is known to depend on tumor stage and Fuhrman grade classification $(2,4)$. Previous studies have reported that all cases of port-site metastasis following laparoscopic radical resection of renal carcinoma were high-grade tumors (Fuhrman grade $>2$ ), including 1 case presenting with a sarcoma-like lesion whose pathological Fuhrman grade was 4 (31-33). The pathology of case 1 in the present study was confirmed as a high-grade, infiltrative, urothelial cell carcinoma of the left renal pelvis and ureter, which was invading the muscular layer. The pathology of case 2 in the present study was confirmed as a high-grade, infiltrative, urothelial cell carcinoma of the right renal pelvis, which was invading the renal parenchyma. The pathology of 
case 3 in the present study was confirmed as Fuhrman grade 3 renal clear cell carcinoma. Port-site metastasis occurred 7 , 1 and 3 months after laparoscopic surgery in cases 1, 2 and 3, respectively. All 3 cases of port-site metastasis were high-grade tumors, in which post-operative metastasis developed rapidly with a poor prognosis. The present cases support the hypothesis that port-site metastasis is associated with tumor stage and Fuhrman grade classification.

Local traumatic factors may contribute to implantation metastasis of cancer cells at the port-site (34). A previous study suggested that cancer cells are more likely to implant during the early stage of wound healing, possibly due to the attachment of cancer cell adhesion protein fibers to the wound surface and wound healing (35). In addition, factors promoting wound healing may be able to promote the growth of cancer cells (36). Considering these risk factors, a previous study proposed that correct repair of the peritoneum surrounding the laparoscopic port-site may be able to reduce the risk of tumor metastasis (37). However, in the present 3 cases, the retroperitoneal approach was employed without rupturing the peritoneum; thus, the current study suggests that local traumatic factors may also induce port-site metastasis.

Furthermore, low immune function may also promote tumor metastasis (38). A previous study hypothesized that the host immune function of the patient may be reduced as a result of certain media factors introduced during the perioperative period (38). Media factors may include the use of anesthetic agents, surgical trauma, transfusion, temperature change, pain and psychological stress $(38,39)$. Previous studies on animal models have demonstrated that surgical trauma may reduce the activity of natural killer cells, thus promoting tumor metastasis $(38,40)$. Furthermore, 3 cases of port-site metastasis following laparoscopic radical resection of renal carcinoma were reported to present varying degrees of immune function impairment, including chronic renal failure in 1 patient, alcoholic cirrhosis in another patient and diabetes in the third patient $(41,42)$. All 3 patients discussed in the present study suffered from renal syndrome without receiving regular treatment, which may, to a certain extent, have impaired holistic immune function during the perioperative period and promoted tumor metastasis.

The performance of a laparoscopy is associated with a number of additional factors that may provoke metastasis, including the presence of pneumoperitoneum, contamination around the port-site, incomplete tumor resection and the particular method used to remove the specimen (30). A previous study reported that air leakage around the port-site could induces a 'chimney effect', suggesting that continuous air leakage around the port would increase the number of cancer cells at the port-site and promote metastasis (43). With regards to the present cases, all ports were fixed in place and no pneumoperitoneal air leakage was noted. A previous study stated that $\mathrm{CO}_{2}$ could stimulate the growth of tumor cells, directly act on tumor cells or interfere with the local defense mechanism (44). In addition, repeated pulling and insertion of the instrument contaminated by tumor rupture during the laparoscopic procedure may result in transfer and invasion of tumor cells at the surgical site, thus increasing the risk of port-site metastasis (45-47). Injection of povidone-iodine into the incision may reduce the risk of port-site metastasis (48). In the current 3 cases, the tumors were excised completely, and no tumor rupture occurred, as evidenced by cases 2 and 3, whose cut edges were pathologically negative for cancer cells. However, the instruments used in these cases were inserted repeatedly without pretreatment with cytotoxic agents, nor was povidone-iodine used at the port-sites, which may have increased the risk of metastasis. Iwamura et al (7) and Chen et al (9) reported cases of port-site metastasis occurring as a result of not using a specimen bag. Therefore, the majority of subsequent studies considered that the use of specimen bags in laparoscopy could reduce port-site contamination and implantation $(15,49)$. However, in the present study, impermeable specimen bags were utilized in all 3 cases, and the bags were not ruptured during the procedure. Therefore, it may be theorized that the occurrence of port-site metastasis is due to multiple factors.

Certain researchers regard the prognosis of port-site metastasis to be unclear (32). In the current study, case 1 survived for 10 months after the detection of port-site implantation, case 2 survived only for 2 months and case 3 remains in a stable condition at present. Case 1 and 2 were diagnosed as high-grade urothelial cell carcinoma complicated with bladder cancer. Case 1 received GC chemotherapy for the port-site metastasis, whilst case 2 did not receive any chemoradiation therapy, thus the life expectancy of case 2 was shorter than that of case 1. Case 3 was diagnosed with high-grade renal clear cell carcinoma and received sunitinib targeted therapy following detection of the port-site metastasis, although the treatment was discontinued 2 weeks later due to bone marrow suppression. The condition of the patient remains stable at present. However, as the patient has been observed for a relatively short time, further follow-up observation is required. Overall, port-site metastasis is more likely to occur in patients with high-grade renal carcinoma or renal pelvis carcinoma compared with patients with low-grade renal cell carcinoma, and the prognosis is usually poor.

In conclusion, a review of the current literature indicates that the occurrence of port-site metastasis subsequent to laparoscopic radical resection of renal pelvis carcinoma and nephron-sparing surgery is relatively rare, and its cause is multifactorial. Although the exact cause remains unclear, the occurrence of port-site metastasis may be considered attributable to the combination of holistic and local factors. Measures to reduce the occurrence of port-site metastasis include strict abidance to the surgical guidelines for tumor resection, avoidance of air leakage at the port-site, use of impermeable specimen bags to remove the specimen under direct vision, irrigation of the laparoscopic surgery instruments and incisional wound with povidone-iodine when necessary, and enhancement of the body's immunity. Post-operative follow-up observation and examination are recommended, particularly in patients with high-grade renal carcinoma or renal pelvis carcinoma, since timely detection of tumor recurrence or metastasis and subsequent administration of systemic chemotherapy are prerequisite for prolonging the life expectance of patients.

\section{References}

1. Schneider C, Jung A, Reymond MA, Tannapfel A, Balli J, Franklin ME, Hohenberger W and Köckerling F: Efficacy of surgical measures in preventing port-site recurrences in a porcine model. Surg Endosc 15: 121-125, 2001. 
2. Rassweiler J, Tsivian A, Kumar AV, Lymberakis C, Schulze M, Seeman O and Frede T: Oncological safety of laparoscopic surgery for urological malignancy: Experience with more than 1,000 operations. J Urol 169: 2072-2075, 2003.

3. Micali S, Celia A, Bove P, De Stefani S, Sighinolfi MC, Kavoussi LR and Bianchi G: Tumor seeding in urological laparoscopy: An international survey. J Urol 171: 2151-2154, 2004.

4. Kadi N, Isherwood M, Al-Akraa M and Williams S: Port-site metastasis after laparoscopic surgery for urological malignancy: Forgotten or missed. Adv Urol 2012: 609531, 2012.

5. Fentie DD, Barrett PH and Taranger LA: Metastatic renal cell cancer after laparoscopic radical nephrectomy: Long-term follow-up. J Endourol 14: 407-411, 2000.

6. Castilho LN, Fugita OE, Mitre AI and Arap S: Port site tumor recurrences of renal cell carcinoma after videolaparoscopic radical nephrectomy. J Urol 165: 519, 2001.

7. Iwamura M, Tsumura H, Matsuda D, Kurosaka S, Yoshida K and Baba S: Port site recurrence of renal cell carcinoma following retroperitoneoscopic radical nephrectomy with manual extraction without using entrapment sac or wound protector. J Urol 171: 1234-1235, 2004.

8. Landman J and Clayman RV: Re: Port site tumor recurrences of renal cell carcinoma after videolaparoscopic radical nephrectomy. J Urol 166: 629-630, 2001.

9. Chen YT, Yang SS, Hsieh CH and Wang CC: Hand port-site metastasis of renal-cell carcinoma following hand-assisted laparoscopic radical nephrectomy: Case report. J Endourol 17: 771-775, 2003.

10. Dhobada S, Patankar S and Gorde V: Case report: Port-site metastasis after laparoscopic radical nephrectomy for renal-cell carcinoma. J Endourol 20: 119-122, 2006.

11. Castillo OA, Vitagliano G, Díaz M and Sánchez-Salas R Port-site metastasis after laparoscopic partial nephrectomy: Case report and literature review. J Endourol 21: 404-407, 2007

12. Greco F, Wagner S, Reichelt O, Inferrera A, Lupo A, Hoda RM, Hamza A and Fornara P: Huge isolated port-site recurrence after laparoscopic partial nephrectomy: A case report. Eur Urol 56 : 737-739, 2009.

13. Masterson TA and Russo P: A case of port-site recurrence and locoregional metastasis after laparoscopic partial nephrectomy. Nat Clin Pract Urol 5: 345-349, 2008.

14. Lee BR, Tan BJ and Smith AD: Laparoscopic port site metastases: Incidence, risk factors, and potential preventive measures. Urology 65: 639-644, 2005

15. Curet MJ: Port site metastases. Am J Surg 187: 705-712, 2004

16. Clayman RV, Kavoussi LR, Soper NJ, Dierks SM, Merety KS Darcy MD, Long SR, Roemer FD, Pingleton ED and Thomson PG: Laparoscopic nephrectomy. N Engl J Med 324: 1370-1371, 1991

17. Stewart GD and Tolley DA: What are the oncological risks of minimal access surgery for the treatment of urinary tract cancer? Eur Urol 46: 415-420, 2004.

18. Castillo OA and Vitagliano G: Port site metastasis and tumor seeding in oncologic laparoscopic urology. Urology 71: 372-378, 2008.

19. Highshaw RA, Vakar-Lopez F, Jonasch E, Yasko AW and Matin SF: Port-site metastasis: the influence of biology. Eur Urol 47: 357-360, 2005.

20. Eng MK, Katz MH, Bernstein AJ, Shikanov S, Shalhav AL and Zorn KC: Laparoscopic port-site metastasis in urologic surgery. J Endourol 22: 1581-1585, 2008.

21. Fuhrman SA, Lasky LC and Limas C: Prognostic significance of morphologic parameters in renal cell carcinoma. Am J Surg Pathol 6: 655-663, 1982

22. Greene FL, Kercher KW, Nelson H, Teigland CM and Boller AM: Minimal access cancer management. CA Cancer J Clin 57: 130-146, 2007.

23. Rajan P and Turna B: New trends in minimally invasive urological surgery. Int Braz J Urol 35: 514-520, 2009.

24. Yu HY, Friedlander DF, Patel S and Hu JC: The current status of robotic oncologic surgery. CA Cancer J Clin 63: 45-56, 2013.

25. Yu HY, Hevelone ND, Lipsitz SR, Kowalczyk KJ and Hu JC: Use costs and comparative effectiveness of robotic assisted, laparoscopic and open urological surgery. J Urol 187: 1392-1398, 2012.

26. Fairey AS, Kassouf W, Estey E, Tanguay S, Rendon R, Bell D, Izawa J, Chin J, Kapoor A, Matsumoto E, et al: Comparison of oncological outcomes for open and laparoscopic radical nephroureterectomy: Results from the Canadian Upper Tract Collaboration. BJU Int 112: 791-797, 2013.
27. Stolla V, Rossi D, Bladou F, Rattier C, Ayuso D and Serment G: Subcutaneous metastases after coelioscopic lymphadenectomy for vesical urothelial carcinoma. Eur Urol 26: 342-343, 1994.

28. Wexner SD and Cohen SM: Port site metastases after laparoscopic colorectal surgery for cure of malignancy. Br J Surg 82: 295-298, 1995

29. Paolucci V, Schaeff B, Schneider M and Gutt C: Tumor seeding following laparoscopy: International survey. World J Surg 23: 989-997, 1999.

30. Ramirez PT, Wolf JK and Levenback C: Laparoscopic port-site metastases: etiology and prevention. Gynecol Oncol 91: 179-189, 2003.

31. Minardi D, Lucarini G, Mazzucchelli R, Milanese G, Natali D, Galosi AB, Montironi R, Biagini G and Muzzonigro G: Prognostic role of Fuhrman grade and vascular endothelial growth factor in pT1a clear cell carcinoma in partial nephrectomy specimens. J Urol 174: 1208-1212, 2005.

32. Stephenson AJ, Chetner MP, Rourke K, Gleave ME, Signaevsky M, Palmer B, Kuan J, Brock GB and Tanguay S: Guidelines for the surveillance of localized renal cell carcinoma based on the patterns of relapse after nephrectomy. J Urol 172: 58-62, 2004.

33. Pearlstone DB, Feig BW and Mansfield PF: Port site recurrences after laparoscopy for malignant disease. Semin Surg Oncol 16: 307-312, 1999.

34. Lee SW, Whelan RL, Southall JC and Bessler M: Abdominal wound tumor recurrence after open and laparoscopic-assisted splenectomy in a murine model. Dis Colon Rectum 41: 824-831, 1998.

35. Murthy SM, Goldschmidt RA, Rao LN, Ammirati M, Buchmann T and Scanlon EF: The influence of surgical trauma on experimental metastasis. Cancer 64: 2035-2044, 1989.

36. Hofer SO, Molema G, Hermens RA, Wanebo HJ, Reichner JS and Hoekstra HJ: The effect of surgical wounding on tumour development. Eur J Surg Oncol 25: 231-243, 1999.

37. Aoki Y, Shimura H, Li H, Mizumoto K, Date K and Tanaka M: A model of port-site metastases of gallbladder cancer: The influence of peritoneal injury and its repair on abdominal wall metastases. Surgery 125: 553-559, 1999.

38. Vallejo R, Hord ED, Barna SA, Santiago-Palma J and Ahmed S Perioperative immunosuppression in cancer patients. J Environ Pathol Toxicol Oncol 22: 139-146, 2003

39. Snyder GL and Greenberg S: Effect of anaesthetic technique and other perioperative factors on cancer recurrence. Br J Anaesth 105: 106-115, 2010.

40. Eggermont AM, Steller EP and Sugarbaker PH: Laparotomy enhances intraperitoneal tumor growth and abrogates the antitumor effects of interleukin-2 and lymphokine-activated killer cells. Surgery 102: 71-78, 1987.

41. Wakim-Fleming J and Mullen KD: Long-term management of alcoholic liver disease. Clin Liver Dis 9: 135-149, 2005.

42. Contin C, Pitard V, Delmas Y, Pelletier N, Defrance T, Moreau JF, Merville P and Déchanet-Merville J: Potential role of soluble CD40 in the humoral immune response impairment of uraemic patients. Immunology 110: 131-140, 2003.

43. Kazemier G, Bonjer HJ, Berends FJ and Lange JF: Port site metastases after laparoscopic colorectal surgery for cure of malignancy. Br J Surg 82: 1141-1142, 1995.

44. Jacobi CA, Sabat R, Böhm B, Zieren HU, Volk HD and Müller JM: Pneumoperitoneum with carbon dioxide stimulates growth of malignant colonic cells. Surgery 121: 72-78, 1997.

45. Gutt CN, Riemer V, Kim ZG, Jacobi CA, Paolucci V and Lorenz M: Impact of laparoscopic colonic resection on tumour growth and spread in an experimental model. Br J Surg 86: $1180-1184,1999$

46. Lee SW, Gleason NR, Bessler M and Whelan RL: Port site tumor recurrence rates in a murine model of laparoscopic splenectomy decreased with increased experience. Surg Endosc 14: 805-811, 2000.

47. Lee SW, Southall J, Allendorf J, Bessler M and Whelan RL: Traumatic handling of the tumor independent of pneumoperitoneum increases port site implantation rate of colon cancer in a murine model. Surg Endosc 12: 828-834, 1998

48. Wittich P, Mearadji A, Marquet RL and Bonjer HJ: Irrigation of port sites: Prevention of port site metastases? J Laparoendosc Adv Surg Tech A 14: 125-129, 2004.

49. Iavazzo C and Gkegkes ID: Port-site metastases in patients with gynecological cancer after robot-assisted operations. Arch Gynecol Obstet 292: 263-269, 2015. 